\title{
Interacting Anyons in Topological Quantum Liquids: The Golden Chain
}

\author{
Adrian Feiguin, ${ }^{1}$ Simon Trebst, ${ }^{1}$ Andreas W. W. Ludwig, ${ }^{2}$ Matthias Troyer, ${ }^{3}$ \\ Alexei Kitaev, ${ }^{1,4}$ Zhenghan Wang, ${ }^{1}$ and Michael H. Freedman ${ }^{1}$ \\ ${ }^{1}$ Microsoft Research, Station Q, University of California, Santa Barbara, California 93106, USA \\ ${ }^{2}$ Physics Department and Kavli Institute for Theoretical Physics, University of California, Santa Barbara, California 93106, USA \\ ${ }^{3}$ Theoretische Physik, Eidgenössische Technische Hochschule Zürich, 8093 Zürich, Switzerland \\ ${ }^{4}$ California Institute of Technology, Pasadena, California 91125, USA
}

(Received 19 December 2006; published 20 April 2007)

\begin{abstract}
We discuss generalizations of quantum spin Hamiltonians using anyonic degrees of freedom. The simplest model for interacting anyons energetically favors neighboring anyons to fuse into the trivial ("identity") channel, similar to the quantum Heisenberg model favoring neighboring spins to form spin singlets. Numerical simulations of a chain of Fibonacci anyons show that the model is critical with a dynamical critical exponent $z=1$, and described by a two-dimensional (2D) conformal field theory with central charge $c=\frac{7}{10}$. An exact mapping of the anyonic chain onto the $2 \mathrm{D}$ tricritical Ising model is given using the restricted-solid-on-solid representation of the Temperley-Lieb algebra. The gaplessness of the chain is shown to have topological origin.
\end{abstract}

DOI: 10.1103/PhysRevLett.98.160409

Non-Abelian anyons are exotic particles expected to exist in certain fractional quantum Hall $(\mathrm{FQH})$ states $[1,2]$. A set of several anyons supports very robust collective states that are degenerate to exponential precision; such states can potentially be used as quantum memory and for quantum computation [3]. However, this degeneracy can be lifted by a short-range interaction if the anyons are very close to each other. As a first step towards understanding interacting anyons, we describe a simple, exactly solvable model that is an anyonic analogue of the quantum Heisenberg chain.

We start by considering the well-known Moore-Read state [1], a candidate state, exhibiting non-Abelian statistics, for the topological nature of $\mathrm{FQH}$ liquids at filling fraction $\nu=\frac{5}{2}$. It has two important types of excitations: quasiholes with electric charge $e / 4$ and neutral fermions. Quasiholes may be trapped by an impurity potential while the fermions can still tunnel between them [4]. For a onedimensional (1D) array of trapped quasiholes, the Hamiltonian can be described in terms of free Majorana fermions on a lattice, which is in turn equivalent to the 1D transverse field Ising model at the quantum phase transition point. The more interesting model discussed here is based on so-called "Fibonacci anyons," which represent the nonAbelian part of the quasiparticle statistics in the $k=3$, $Z_{k}$-parafermion state [2], an effective theory for $\mathrm{FQH}$ liquids at filling fraction $\nu=\frac{12}{5}$ [5]. Even without parameter fine-tuning, these 1D anyonic arrays will be shown to exhibit gapless excitations due to topological symmetry.

Model. - Our model describes pairwise interactions within an array of $L$ anyons, for instance along a chain as shown in Fig. 1(a). In the Fibonacci theory there are only two types of particles: the Fibonacci anyon, denoted by $\tau$, and the trivial particle denoted by 1 with a fusion rule $\tau \times$ $\tau=1+\tau$. We refer to the label 1 or $\tau$ as the topological charge. When two neighboring anyons interact, indicated
PACS numbers: 05.30.Pr, 03.65.Vf, 73.43.Lp

in the figure by the ellipses, they can either fuse in the trivial channel, annihilating each other, or in the nontrivial one, becoming a single $\tau$ anyon [6]. We define our model by assigning an energy gain if they fuse along the trivial channel. This is an anyonic analogue of the spin- $\frac{1}{2}$ quantum Heisenberg antiferromagnet, which assigns an energy gain to two neighboring spin- $\frac{1}{2}$ fusing into a spin-0 singlet as compared to a spin-1 triplet.

To define the Hilbert space we consider the treelike fusion diagram in Fig. 1(b). The basis corresponds to all admissible labelings $\left|x_{1}, x_{2}, \ldots\right\rangle$ of the links, with $x_{i}=1$ or $\tau$. Each label represents the combined topological charge of the particles left to a given point. Not all possible values $\left(x_{1}, x_{2}, \ldots\right)$ represent allowed basis states due to the fusion rules: a 1 must always be preceded and followed by a $\tau$, since the fusion of a 1 and a $\tau$ always gives a $\tau$. This reduces the dimension of the Hilbert space of the open chain (with $\tau$ labels at the boundary) to the Fibonnacci sequence $\operatorname{dim}_{L}=F_{L+1}$, and for the periodic chain $\operatorname{dim} L=F_{L-1}+F_{L+1}$. For large $L$ it is well known that

a)

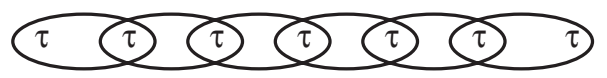

b)

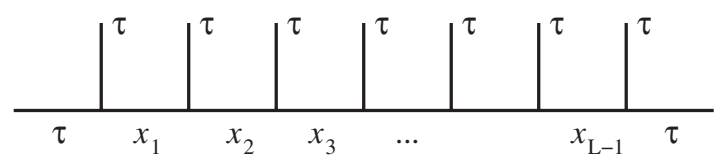

c)

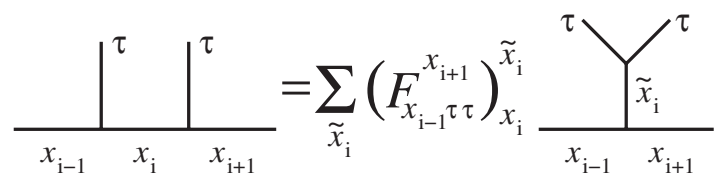

FIG. 1. (a) Illustration of the Fibonacci chain with $L \tau$-anyons. (b) The fusion path. (c) Definition of the $F$ matrix. 
these numbers grow at a rate $\operatorname{dim}_{L} \propto \varphi^{L}$, where $\varphi=(1+$ $\sqrt{5}) / 2$ is the golden ratio. This Hilbert space has no natural decomposition in the form of a tensor product of single-site states, in contrast to $S U(2)$ quantum spin chains.

To generate a local Hamiltonian $\mathbf{H}=\sum_{i} \mathbf{H}_{i}$ assigning an energy to the fusion of two neighboring $\tau$ anyons we use the so-called $F$ matrix to transform the local basis as shown in Fig. 1(c). In the transformed basis the state $\tilde{x}_{i}$ corresponds to the fusion of the two anyons. The Hamiltonian is then defined by assigning an energy $E_{\tau}=0$ for $\tilde{x}_{i}=\tau$, and $E_{1}=-1$ for $\tilde{x}_{i}=1$. The resulting local terms $\mathbf{H}_{i}$ contain three-body interactions in the link basis,

$$
\begin{aligned}
\mathbf{H}_{i}\left|x_{i-1} x_{i} x_{i+1}\right\rangle & =\sum_{x_{i}^{\prime}=1, \tau}\left(\mathbf{H}_{i}\right)_{x_{i}}^{x_{i}^{\prime}}\left|x_{i-1} x_{i}^{\prime} x_{i+1}\right\rangle \\
\text { with }\left(\mathbf{H}_{i}\right)_{x_{i}}^{x_{i}^{\prime}} & =-\left(F_{x_{i-1} \tau \tau}^{x_{i+1}}\right)_{x_{i}}^{1}\left(F_{x_{i-1} \tau \tau}^{x_{i+1}}\right)_{x_{i}^{\prime}}^{1}
\end{aligned}
$$

It is diagonal in the subspace $\left\{\left|x_{i-1} x_{i} x_{i+1}\right\rangle\right\}=$ $\{|1 \tau 1\rangle,|1 \tau \tau\rangle,|\tau \tau 1\rangle\}, \mathbf{H}_{i}=\operatorname{diag}\{-1,0,0\}$, where the $F$ matrix is a number due to the constraints arising from the fusion rules. For the case $x_{i-1}=x_{i+1}=\tau$, the $F$ matrix and the corresponding Hamiltonian are the following $2 \times 2$ matrices $\left(x_{i}, x_{i}^{\prime} \in\{1, \tau\}\right)$

$$
\mathbf{F}_{\tau \tau \tau}^{\tau}=\left(\begin{array}{cc}
\varphi^{-1} & \varphi^{-1 / 2} \\
\varphi^{-1 / 2} & -\varphi^{-1}
\end{array}\right), \quad \mathbf{H}_{i}=-\left(\begin{array}{cc}
\varphi^{-2} & \varphi^{-3 / 2} \\
\varphi^{-3 / 2} & \varphi^{-1}
\end{array}\right)
$$

The Hamiltonian can be written in terms of Pauli matrices:

$$
\begin{aligned}
\mathbf{H}_{i}= & \left(n_{i-1}+n_{i+1}-1\right) \\
& -n_{i-1} n_{i+1}\left(\varphi^{-3 / 2} \sigma_{i}^{x}+\varphi^{-3} n_{i}+1+\varphi^{-2}\right),
\end{aligned}
$$

where the sum runs over the links of the chain. In this expression, the operators $n_{i}$ count the $\tau$-particle occupation on link $i, n_{i}=\frac{1}{2}\left(1-\sigma_{i}^{z}\right)=0,1$, and the Hamiltonian $\mathbf{H}$ acts on the constrained Hilbert space defined above.

Central charge. - We simulated this model numerically, and calculated the finite-size gap by exact diagonalization using the ALPS libraries [7]. A finite-size analysis shows that the gap vanishes linearly in $\frac{1}{L}$, indicative of a critical model with dynamical critical exponent $z=1$ described by a conformal field theory (CFT). The central charge $c$ of a CFT can be calculated from the finite-size scaling of the entanglement entropy [8]. A density matrix renormalization group method [9] calculation gives a central charge estimate of $c=0.701 \pm 0.001$. Since possible (unitary) CFTs in the vicinity of these estimates have central charges [10] $\frac{1}{2}, \frac{7}{10}$, or $\frac{4}{5}$ we can unambiguously conclude that our results are consistent only with central charge $c=\frac{7}{10}$.

Mapping and exact solution. - We now proceed to derive these results exactly. By construction $\frac{1}{\varphi} \mathbf{X}_{i}=-\mathbf{H}_{i}$ is a projector onto the trivial particle. One can then verify that the operators $\mathbf{X}_{i}$ form a representation of the TemperleyLieb (TL) algebra [11]

$$
\begin{gathered}
\left(\mathbf{X}_{i}\right)^{2}=d \mathbf{X}_{i}, \quad \mathbf{X}_{i} \mathbf{X}_{i \pm 1} \mathbf{X}_{i}=\mathbf{X}_{i} \\
{\left[\mathbf{X}_{i}, \mathbf{X}_{j}\right]=0 \quad \text { for }|i-j| \geq 2}
\end{gathered}
$$

where the " $d$-isotopy" parameter equals the golden ratio, $d=\varphi$. This representation can be seen to be identical to the standard TL algebra representation associated with $S U(2)_{k}$ at level $k=3$. For an arbitrary integer $k>0$, the latter contains $k+1$ anyon species labeled by $j=$ $0, \frac{1}{2}, 1, \ldots, \frac{k}{2}$, satisfying the fusion rules of $S U(2)_{k}[12]$. The operators $\mathbf{e}_{i}$ defined by

$$
\begin{aligned}
\mathbf{e}[i]\left|j_{i-1} j_{i} j_{i+1}\right\rangle & =\sum_{j_{i}^{\prime}}\left(\mathbf{e}[i]_{j_{i-1}}^{j_{i+1}}\right)_{j_{i}}^{j_{j}^{\prime}}\left|j_{i-1} j_{i}^{\prime} j_{i+1}\right\rangle \\
\text { and } \quad\left(\mathbf{e}[i]_{j_{i-1}}^{j_{i+1}}\right)_{j_{i}}^{j_{i}^{\prime}} & =\delta_{j_{i-1}, j_{i+1}} \sqrt{\frac{S_{j_{i}}^{0} S_{j_{i}^{\prime}}^{0}}{S_{j_{i-1}}^{0} S_{j_{i+1}}^{0}}}
\end{aligned}
$$

are known [13] to form a representation of the TemperleyLieb algebra (3) for any value of $k$, where $\left|j_{i}-j_{i+1}\right|=\frac{1}{2}$ and $S_{j}^{j^{\prime}}:=\sqrt{\frac{2}{(k+2)}} \sin \left[\pi \frac{(2 j+1)\left(2 j^{\prime}+1\right)}{k+2}\right][14]$.

Our model can be cast into this form at $k=3$ by first mapping $x_{i}=1 \rightarrow j_{i}=0$, and $x_{i}=\tau \rightarrow j_{i}=1$, and then applying the $S U(2)_{3}$ fusion rule $\frac{3}{2} \times j=\frac{3}{2}-j$ to the evennumbered sites. This maps any admissible labeling $\left|x_{1}, x_{2}, \ldots\right\rangle$ uniquely into $\left|j_{1}, j_{2}, \ldots\right\rangle$ where for oddnumbered sites $j_{2 i+1} \in\{0,1\}$, and for even-numbered sites $j_{2 i} \in\left\{\frac{1}{2}, \frac{3}{2}\right\}$. This relabeling maps the matrix elements of $\mathbf{X}_{i}$ into those of $\mathbf{e}_{i}$ from Eq. (4).

The Hamiltonian in Eq. (1) is that corresponding to a standard (integrable) lattice model description of the classical 2D tricritical Ising model, known as the restrictedsolid-on-solid (RSOS) model [15]. Specifically, the tworow transfer matrix $\mathbf{T}:=\mathbf{T}_{2} \mathbf{T}_{1}$ of this lattice model, see Fig. 2, is written in terms of Boltzmann weights $\mathbf{W}[i]$ assigned to a plaquette $i$ of the square lattice

$$
\begin{gathered}
\mathbf{T}_{1}:=\prod_{n} \mathbf{W}[2 n] \quad \text { and } \quad \mathbf{T}_{2}:=\prod_{n} \mathbf{W}[2 n+1] \text {, } \\
\text { with } \quad \mathbf{W}[i]_{\vec{j}}^{\overrightarrow{j^{\prime}}}=\frac{\sin \left[\frac{\pi}{k+2}-u\right]}{\sin \frac{\pi}{k+2}} \mathbf{1}_{\vec{j}}^{\vec{j}^{\prime}}+\frac{\sin u}{\sin \frac{\pi}{k+2}} \mathbf{e}[i]_{\vec{j}}^{\vec{j}^{\prime}},
\end{gathered}
$$

where $\vec{j}=\left(j_{1}, j_{2}, \ldots\right)$. The parameter $u>0$ is a measure of the lattice anisotropy, $\mathbf{1}$ is the identity operator, and

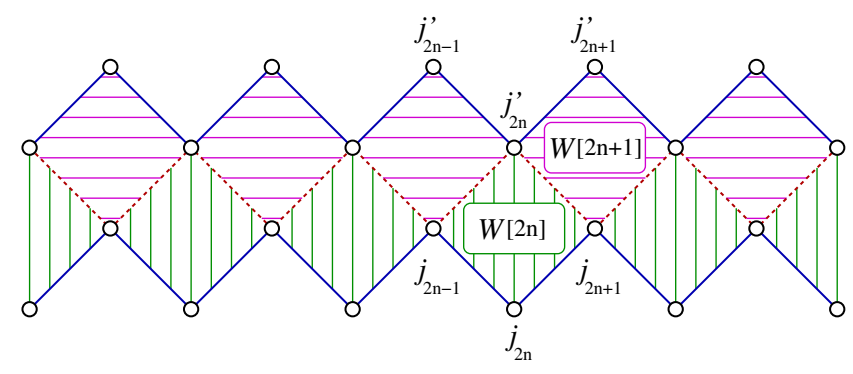

FIG. 2 (color online). Transfer matrix of the RSOS model. 


$$
\mathbf{e}[i]_{\vec{j}}^{\vec{j}^{\prime}}:=\left[\prod_{m \neq i} \delta_{j_{m}^{\prime}, j_{m}}\right]\left(\mathbf{e}[i]_{j_{i-1}}^{j_{i+1}}\right)_{j_{i}}^{j_{i}^{\prime}}
$$

The Hamiltonian of the so-defined lattice model is obtained from its transfer matrix by taking, as usual [16], the extremely anisotropic limit, $u \ll 1$,

$$
\begin{aligned}
& \mathbf{T}=\exp \left\{-a\left(\mathbf{H}+c_{1}\right)+O\left(a^{2}\right)\right\}, \\
& a=\frac{u \varphi}{\sin [\pi /(k+2)]} \ll 1
\end{aligned}
$$

yielding $\mathbf{H}=-\sum_{i} \frac{1}{\varphi} \mathbf{e}_{i}$ ( $c_{1}$ is an unimportant constant). Since the operators $\mathbf{X}_{i}$ can be identified with $\mathbf{e}_{i}$, this demonstrates that the Hamiltonian of the Fibonacci chain is exactly that of the corresponding $k=3$ RSOS model which is a lattice description of the tricritical Ising model at its critical point. The latter is a well-known (supersymmetric) CFT with central charge $c=\frac{7}{10}[17,18]$. Analogously one obtains [19] for general $k$ the $(k-1)^{\text {st }}$ unitary minimal CFT [10] of central charge $c=1-6 /(k+1)(k+2)$. A ferromagnetically coupled Fibonacci chain (energetically favoring the fusion along the $\tau$ channel) is described [20] by the critical 3-state Potts model with $c=\frac{4}{5}$ and, for general $k$, by the critical $Z_{k}$-parafermion CFT $[15,19]$ with central charge $c=2(k-1) /(k+2)$.

Excitation spectra. - We calculated the excitation spectra of chains up to size $L=37$ with open and periodic boundary conditions using exact diagonalization, as shown in Fig. 3. The numerical results not only confirm the CFT predictions but also reveal some important details about the correspondence between continuous fields and microscopic observables. In general, low-energy states on a ring are associated with local conformal fields [21], whose holomorphic and antiholomorphic parts belong to representations of the Virasoro algebra, described by conformal weights $h_{L}$ and $h_{R}$. The energy levels are given by

$$
E=E_{1} L+\frac{2 \pi v}{L}\left(-\frac{c}{12}+h_{L}+h_{R}\right),
$$

corresponding to states with a choice of momenta $K=$ $h_{L}-h_{R}$ or $K=h_{L}-h_{R}+L / 2$ in units of $2 \pi / L$, where $E_{1}, v$ are nonuniversal constants. Here, $h_{L}=h_{L}^{(0)}+m_{L}$ and $h_{R}=h_{R}^{(0)}+m_{R}$, where $h_{L}^{(0)}, h_{R}^{(0)}$ correspond to weights of "primary" fields and $m_{L}$ and $m_{R}$ are non-negative integers describing so-called "descendant" fields. The numerical spectra for even values of $L$ (see the first plot in Fig. 3) agree with Eq. (7), exhibiting primary fields with $h_{L}^{(0)}=h_{R}^{(0)}=0, \frac{1}{10}, \frac{3}{5}, \frac{3}{2}, \frac{3}{80}, \frac{7}{16}$, which are conventionally denoted by $I, \varepsilon, \varepsilon^{\prime}, \varepsilon^{\prime \prime}, \sigma, \sigma^{\prime}$, respectively [22]. The momenta of the last two fields and their descendants are near $K=L / 2$, as compared to the other four, indicating that the corresponding microscopic observables have alternating sign on the lattice. Such "staggered" fields must have nontrivial monodromy with respect to a space-time dislocation (i.e., the insertion or removal of a site at some particular time). Such a dislocation is characterized by a chiral $\varepsilon^{\prime \prime}$ field, say, $\varepsilon_{L}^{\prime \prime}$ [23]. The log of the monodromy
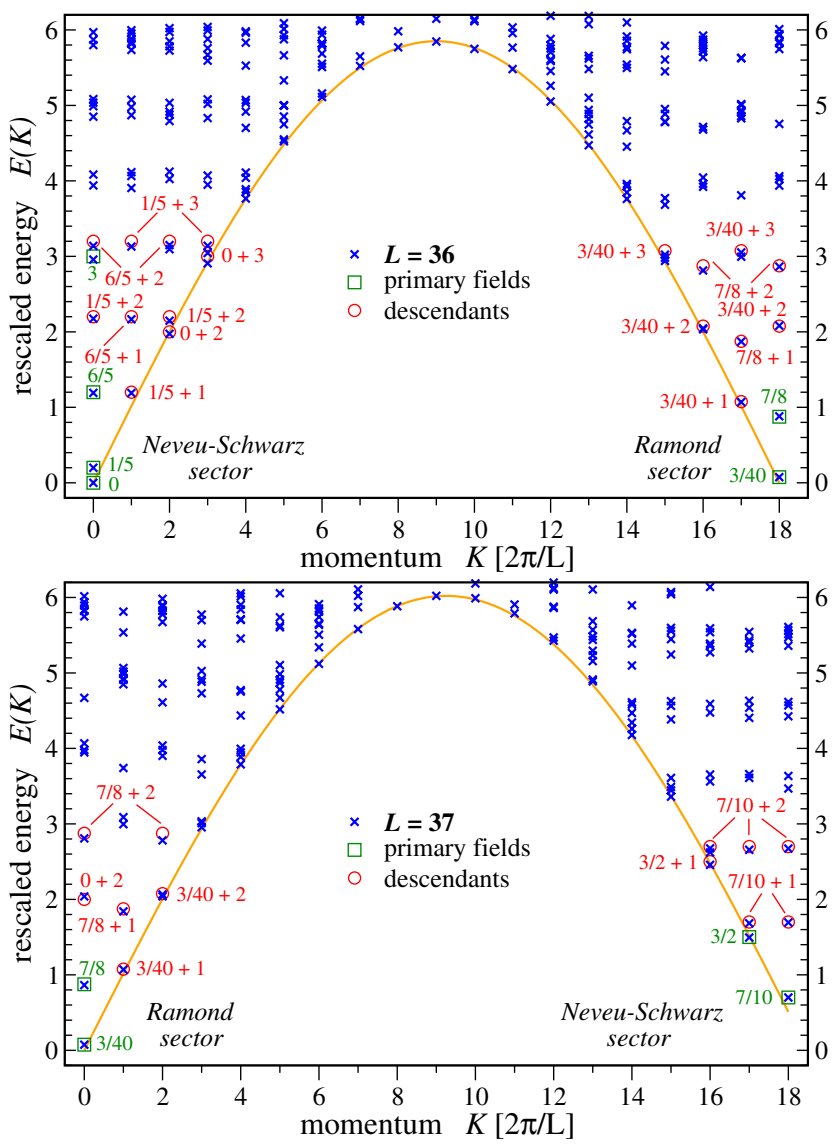

FIG. 3 (color online). Energy spectra for periodic chains of size $L$. Energies are rescaled and shifted such that the two lowest eigenvalues match the CFT assignments. Open boxes indicate positions of primary fields of the $c=\frac{7}{10} \mathrm{CFT}$. Open circles give positions of descendant fields as indicated. As a guide to the eye the solid line is a cosine-fit of the dispersion.

factor $\exp \left[2 \pi i\left(h_{L}^{\psi \times \varepsilon^{\prime \prime}}-h_{L}^{\psi}-h_{L}^{\varepsilon^{\prime \prime}}\right)\right]$ matches the momenta $K$ in Fig. 3 [24]. Given this information, we may predict that the states of an odd size ring are associated with fields of the form $\xi_{L} \eta_{R}$, where $\eta=\xi \times \varepsilon^{\prime \prime}$. These include six primary fields, $\varepsilon_{L}^{\prime \prime}, \varepsilon_{R}^{\prime \prime}, \varepsilon_{L}^{\prime} \varepsilon_{R}, \varepsilon_{L} \varepsilon_{R}^{\prime}, \sigma_{L} \sigma_{R}, \sigma_{L}^{\prime} \sigma_{R}^{\prime}$, as well as their descendants. Integrality of the momentum $K$ dictates the choice of $K$ [see below Eq. (7)], as in Fig. 3.

For open boundary conditions the spectra are known to be described by, say, the holomorphic sector only [25]. To explain the numerical data, we need to assume that the ends of the chain are charaterized by a boundary field $\varepsilon$ (or equally well $\varepsilon^{\prime}$ ). Thus, for an even size $L$ the spectrum is described by $\varepsilon \times \varepsilon=I+\varepsilon^{\prime}$ (plus descendants). For an odd size, this result is to be modified by fusion with $\varepsilon^{\prime \prime}$, yielding $\varepsilon^{\prime \prime}+\varepsilon$. These predictions agree excellently with the numerics in Table I.

Hidden symmetries. - The critical behavior of our model is not just a peculiarity of the exact solution but rather has topological origin. In general, an effective lowenergy Lagrangian admits perturbations of the form $\int \psi(x, \tau) d x d \tau$, where $\psi$ may be any local field that is 
TABLE I. Lowest eigenvalues for open Fibonacci chains of size $L$. The two lowest eigenvalues are rescaled and shifted such that they match the conformal field theory assignments.

\begin{tabular}{ccccc}
\hline \hline Eigenvalue & $\begin{array}{c}\text { Nnumerics } \\
L=31\end{array}$ & $\begin{array}{c}\text { CFT } \\
\text { assignment }\end{array}$ & $\begin{array}{c}\text { Numerics } \\
L=32\end{array}$ & $\begin{array}{c}\text { CFT } \\
\text { assignment }\end{array}$ \\
\hline 0 & 0.10 & $\frac{1}{10}$ & 0 & 0 \\
1 & 1.10 & $\frac{1}{10}+1$ & 0.60 & $\frac{3}{5}$ \\
2 & 1.49 & $\frac{3}{2}$ & 1.60 & $\frac{3}{5}+1$ \\
3 & 2.09 & $\frac{1}{10}+2$ & 2.02 & $0+2$ \\
4 & 2.47 & $\frac{3}{2}+1$ & 2.58 & $\frac{3}{5}+2$ \\
5 & 3.07 & $\frac{1}{10}+3$ & 2.59 & $\frac{3}{5}+2$ \\
6 & 3.11 & $\frac{1}{10}+3$ & 3.01 & $0+3$ \\
7 & 3.44 & $\frac{3}{2}+2$ & 3.56 & $\frac{3}{5}+3$ \\
8 & 3.46 & $\frac{3}{2}+2$ & 3.56 & $\frac{3}{5}+3$ \\
\hline \hline
\end{tabular}

consistent with all applicable symmetries. Such terms are relevant if $h_{L}^{\psi}+h_{R}^{\psi}<2$, in which case they may open a spectral gap or induce crossover to different critical behavior at large distances. In the tricritical Ising model, there are four relevant fields: $\varepsilon_{L} \varepsilon_{R}, \varepsilon_{L}^{\prime} \varepsilon_{R}^{\prime}, \sigma_{L} \sigma_{R}, \sigma_{L}^{\prime} \sigma_{R}^{\prime}$. Some explanation is in order as to why these fields do not appear in the effective Lagrangian of our model. The fields $\sigma$ and $\sigma^{\prime}$ are staggered and thus prohibited by translational symmetry. Excluding $\varepsilon$ and $\varepsilon^{\prime}$ requires a more subtle argument. The Fibonacci ring has a topological symmetry, which corresponds to adding an extra $\tau$-line parallel to the spine of the fusion diagram [Fig. 1(b)] and merging it with the diagram using the $F$ matrix. We denote this operator by $Y$.

$$
\left\langle x_{0}^{\prime}, \ldots, x_{L-1}^{\prime}|Y| x_{0}, \ldots, x_{L-1}\right\rangle=\prod_{i=0}^{L-1}\left(F_{\tau x_{i}}^{x_{i+1}^{\prime}}\right)_{x_{i+1}}^{x_{i}^{\prime}}
$$

where the identification $L \equiv 0$ is used. We may think of the fusion diagram as a description of a process that generates a set of $\tau$ anyons on a circle from the local vacuum. Then $Y$ describes another particle moving along the circle, inside or outside. The operator $Y$ is sensitive to a possible topological charge $y=1, \tau$ located at the center of the circle. Thus $Y$ has two eigenvalues, $S_{y \tau} / S_{y 1}=\varphi,-\varphi^{-1}$. We conjecture that the low-energy states associated with fields $I, \varepsilon^{\prime \prime}, \sigma^{\prime}$ are in the trivial $(y=1)$ sector, and the fields $\varepsilon^{\prime}, \varepsilon$, $\sigma$ are in the $y=\tau$ sector. In fact, the topological fusion algebra (defined by the rule $\tau \times \tau=1+\tau$ ) is a quotient of the CFT fusion algebra.

We may imagine that the interaction between the anyons alters the topological liquid in which the anyons are excitations, producing an annulus of a different liquid. Some of the local fields correspond to the tunneling of a $\tau$ anyon between the inner and outer edge of the annulus. Such a process is actually forbidden as it would change the topological charge $y$. Thus, only fields in the trivial topological sector $y=1$ are allowed as perturbations. This excludes $\psi=\varepsilon_{L} \varepsilon_{R}$ and $\psi=\varepsilon_{L}^{\prime} \varepsilon_{R}^{\prime}$.

Outlook. - Extensions to chains of anyons in topological liquids corresponding to the non-Abelian statistics of higher members $(k>3)$ of the Read-Rezayi series have been mentioned below Eq. (6), but their topological stability is an open issue. In analogy to quantum spin chains, additional interactions (e.g., dimerization or coupling two chains) lead to interesting physics. Disordered anyonic chains are currently being investigated [26]. For 2D anyonic structures gapless phases of non-Fermi liquid type might potentially also emerge.

We thank E. Ardonne, N. Bonesteel, P. Fendley, C. Nayak, G. Refael, S. H. Simon, and J. Slingerland for discussions.

[1] G. Moore and N. Read, Nucl. Phys. B360, 362 (1991).

[2] N. Read and E. Rezayi, Phys. Rev. B 59, 8084 (1999).

[3] A. Yu Kitaev, Ann. Phys. (N.Y.) 303, 2 (2003).

[4] See, e.g., N. Read and A. W. W. Ludwig, Phys. Rev. B 63, 024404 (2000).

[5] J. S. Xia et al., Phys. Rev. Lett. 93, 176809 (2004).

[6] See, e.g., J. Preskill, Lecture Notes on Quantum Computation, http://www.theory.caltech.edu/ preskill/ ph229.

[7] F. Alet et al., J. Phys. Soc. Jpn. Suppl. 74, 30 (2005).

[8] C. Holzhey et al., Nucl. Phys. B424, 443 (1994); P. Calabrese and J. Cardy, J. Stat. Mech. (2004) P06002.

[9] S. R. White, Phys. Rev. Lett. 69, 2863 (1992).

[10] D. Friedan, Z. Qiu, and S. Shenker, Phys. Rev. Lett. 52, 1575 (1984).

[11] N. Temperley and E. Lieb, Proc. R. Soc. A 322, 251 (1971).

[12] D. Gepner and E. Witten, Nucl. Phys. B278, 493 (1986); A. B. Zamolodchikov and V. A. Fateev, Sov. J. Nucl. Phys. 43, 657 (1986).

[13] V. Jones, C. R. Acad. Sci. Paris Sér. I Math. 298, 505 (1984); A. Kuniba, Y. Akutsu, and M. Wadati, J. Phys. Soc. Jpn. 55, 3285 (1986); V. Pasquier, Nucl. Phys. B285, 162 (1987); H. Wenzl, Invent. Math. 92, 349 (1988); For a recent discussion, see P. Fendley, J. Phys. A 39, 15445 (2006).

[14] The matrix $S_{j}^{j^{\prime}}$ is the modular $S$-matrix of $S U(2)_{k}$ CFT.

[15] G. E. Andrews, R. J. Baxter, and P. J. Forrester, J. Stat. Phys. 35, 193 (1984).

[16] See, for example, R. J. Baxter, Exactly Solved Models in Statistical Mechanics (Academic Press, London, 1982).

[17] D. Friedan, Z. Qiu, and S. Shenker, Phys. Lett. B 151, 37 (1985).

[18] A. A. Belavin, A. M. Polyakov, and A. B. Zamolodchikov, Nucl. Phys. B241, 333 (1984).

[19] D. A. Huse, Phys. Rev. B 30, 3908 (1984).

[20] In this case the sign of $u$ in Eq. (5) is reversed.

[21] J. L. Cardy, J. Phys. A 17, L385 (1984); Nucl. Phys. B270, 186 (1986).

[22] The last two fields are the order operators, i.e., those coupling to applied magnetic fields.

[23] $\varepsilon^{\prime \prime}$ is the superpartner of the stress tensor [17].

[24] Fusion with $\varepsilon^{\prime \prime}$ corresponds [17,18] to $I \leftrightarrow \varepsilon^{\prime \prime}, \varepsilon \leftrightarrow \varepsilon^{\prime}$, $\sigma \leftrightarrow \sigma, \sigma^{\prime} \leftrightarrow \sigma^{\prime}$.

[25] J. L. Cardy, Nucl. Phys. B240, 514 (1984).

[26] N.E. Bonesteel and Kun Yang, cond-mat/0612503. 